Research Article

\title{
Composite Compensation Control of Robotic System Subject to External Disturbance and Various Actuator Faults
}

\author{
Hao Sheng and Xia Liu \\ School of Electrical Engineering and Electronic Information, Xihua University, Chengdu 610039, China \\ Correspondence should be addressed to Xia Liu; xliu_uestc@yahoo.com
}

Received 2 May 2020; Revised 24 June 2020; Accepted 27 June 2020; Published 26 July 2020

Guest Editor: Esam Hafez Abdelhameed

Copyright ( $\odot 2020$ Hao Sheng and Xia Liu. This is an open access article distributed under the Creative Commons Attribution License, which permits unrestricted use, distribution, and reproduction in any medium, provided the original work is properly cited.

\begin{abstract}
This paper studies the problems of external disturbance and various actuator faults in a nonlinear robotic system. A composite compensation control scheme consisting of adaptive sliding mode controller and observer-based fault-tolerant controller is proposed. First, a sliding mode controller is designed to suppress the external disturbance, and an adaptive law is employed to estimate the bound of the disturbance. Next, a nonlinear observer is designed to estimate the actuator faults, and a fault-tolerant controller is obtained based on the observer. Finally, the composite compensation control scheme is obtained to simultaneously compensate the external disturbance and various actuator faults. It is proved by Lyapunov function that the disturbance compensation error and fault compensation error can converge to zero in finite time. The theoretical results are verified by simulations. Compared to the conventional fault reconstruction scheme, the proposed control scheme can compensate the disturbance while dealing with various actuator faults. The fault compensation accuracy is higher, and the fault error convergence rate is faster. Moreover, the robot can track the desired position trajectory more accurately and quickly.
\end{abstract}

\section{Introduction}

Robotic system is a complex nonlinear system with the characteristics of multiple variables, high nonlinearity, and strong coupling. In robotic system, there are a variety of problems, such as external disturbance and actuator fault. The position tracking performance of the robot will decrease due to disturbance. Meanwhile, the controller needs to tolerate actuator fault to keep the robotic system stable [1-3]. Therefore, disturbance and actuator fault are two of the main issues to be solved in robot control.

For robotic system with disturbance, sliding mode control has been widely applied due its robustness to disturbance and uncertainty [4]. However, there are some drawbacks in conventional sliding mode control. For example, the error cannot converge in finite time, and there exits chattering phenomenon. In addition, the upper bound of the disturbance needs to be known. In order to avoid the drawbacks in conventional sliding mode control, observer is one of the effective approaches. In [5], a composite controller based on a nonlinear controller and a nonlinear disturbance observer was proposed for nonlinear systems, where the observer was employed to estimate the disturbance generated by an exogenous system. In [6], the external disturbance in a nonlinear system was viewed as an unknown input. An adaptive extended state observer was designed to estimate the unknown input, and then, a controller was designed to compensate the external disturbance using the estimated value. In [7], for the unknown matched and mismatched time-varying disturbances in a robotic system, a continuous sliding mode control based on generalized proportional integral observer was proposed. The observer was to estimate the matched disturbance and mismatched disturbance, respectively. The continuous sliding mode manifold was to remove the offset caused by the mismatched disturbance. In [8], the uncertain hydrodynamics and unknown external disturbance in an underwater robotic system were regarded as a lumped disturbance. An integral sliding mode controller based on extended state observer was presented. The extended state observer was to 
estimate the lumped disturbance and unmeasurable states, and the adaptive gain update algorithm was to estimate the bound of the lumped disturbance. In [9], the model errors, uncertainties, friction, and unknown external disturbances in automobile electrocoating conveying mechanism were all regarded as a lumped disturbance. A nonlinear disturbance observer was to estimate the lumped disturbance, and a sliding mode controller was designed for the hybrid seriesparallel mechanism. Although the approaches in [5-9] can effectively deal with the disturbance in the system, they all potentially assume that all the actuators in the system are working normally without any fault.

In fact, in addition to external disturbance, many mechanical systems and electronic devices, such as sensors, actuators, and amplifiers, may undergo fault due to aging, affecting the performance and even safety of the system $[10-12]$. In order to ensure the performance and safety of the system when actuator fault occurs, different fault-tolerant control schemes have been proposed. In [13], a fault reconstruction scheme based on terminal sliding mode observer and fault-tolerant control was proposed for robotic manipulators. The fault reconstruction error can converge to zero in finite time. Nevertheless, only actuator fault was considered. In [14], for external disturbance and actuator fault in manipulator, a fault-tolerant control based on adaptive dynamic sliding mode was proposed. However, only loss of effectiveness fault was considered. In [15], actuator faults and friction in a robotic system were regarded as total uncertain dynamics. A sliding mode observer was designed to estimate the total uncertain dynamics. A nonlinear observer was used to reconfigure the uncertainty. However, since the fault and friction were regarded as total uncertain dynamics, their respective characteristic cannot be reflected. In [16], actuator faults and external collision in robot manipulator were regarded as centralized disturbance. A sliding mode observer was used to estimate the velocity and centralized disturbance. A protective control framework based on disturbance reconstruction was proposed. Nevertheless, the characteristic of fault was not formally described in [16]. In [17], for robots subject to unmatched disturbance and actuator fault, a fault-tolerant adaptive control based on disturbance observer and backstepping control was proposed. Nevertheless, the disturbance error cannot converge to zero in finite time, and the error convergence rate was slow. In $[18,19]$, for actuator fault, matched or unmatched disturbance in a class of uncertain nonlinear systems, an active fault-tolerant control was designed based on integral-type sliding mode control. However, since active fault-tolerant control was based on fault information, delay of the fault information feedback will result in delay of the fault compensation time. Consequently, the system may become unstable. In [20], actuator fault, external disturbance, and input saturation were regarded as total uncertainty for the robotic system, and a finite-time fault-tolerant adaptive robust control strategy was proposed. The total uncertainty was estimated by the adaptive law, and then, a fault-tolerant adaptive robust controller was obtained by the integral backstepping control. However, as actuator fault, external disturbance, and input saturation in the system were treated as total uncertainty, and their respective characteristic could not be reflected well. Moreover, only time-varying fault was considered in [20].

In this paper, a composite compensation control approach is proposed for a nonlinear robotic system with external disturbance and various actuator faults. The proposed composite compensation controller consists of an adaptive sliding mode controller and an observer-based fault-tolerant controller. Compared to the conventional fault reconstruction scheme, the proposed control can compensate disturbance while dealing with various actuator faults, including no fault, loss of effectiveness fault, and floating around trim fault. The fault compensation accuracy is higher, and the fault error convergence rate is faster. Moreover, the robot can track the desired position trajectory more accurately and quickly.

The remainder of this paper is organized as follows: in Section 2, the model of robotic system subject to external disturbance and actuator faults is formally described; in Section 3, the composite compensation control is designed based on adaptive sliding mode control and observer-based fault-tolerant control, and the convergence of the disturbance compensation error and fault compensation error is proved; simulations are provided in Section 4; and the paper is concluded in Section 5.

\section{Model of Robotic System Subject to External Disturbance and Actuator Faults}

A nonlinear robotic system with external disturbance and actuator faults is considered in this paper, as shown in Figure 1.

2.1. Model of Robotic System Subject to External Disturbance. The dynamic model of a $n$-DOF nonlinear robot subject to external disturbance can be described as follows [21]:

$$
M(q) \ddot{q}+C(q, \dot{q}) \dot{q}+G(q)+\tau_{d}=u,
$$

where $q \in R^{n \times 1}, \dot{q} \in R^{n \times 1}$, and $\ddot{q} \in R^{n \times 1}$ represent the joint position, joint velocity, and joint acceleration of the robot, respectively; $M(q) \in R^{n \times n}, C(q, \dot{q}) \in R^{n \times n}$, and $G(q) \in R^{n \times 1}$ represent the inertia matrix, Coriolis and centrifugal term, and gravity term. $u \in R^{n \times 1}$ is the control torque, and $\tau_{d} \in R^{n \times 1}$ denotes the external disturbance.

In practical applications, the external disturbance of a system is usually bounded [22], i.e.,

$$
\left\|\tau_{d}\right\| \leq F
$$

where $F$ is an unknown constant.

For the dynamic model of the robot (1), there are two important properties.

Property 1. The inertia matrix $M(q)$ is symmetric and positive definite which satisfies

$$
\lambda_{0}\|\xi\|^{2} \leq \xi^{T} M(q) \xi \leq \lambda_{1}\|\xi\|^{2},
$$

where $\lambda_{0}$ and $\lambda_{1}$ are positive constants and $\forall \xi \in R^{n \times 1}$. 


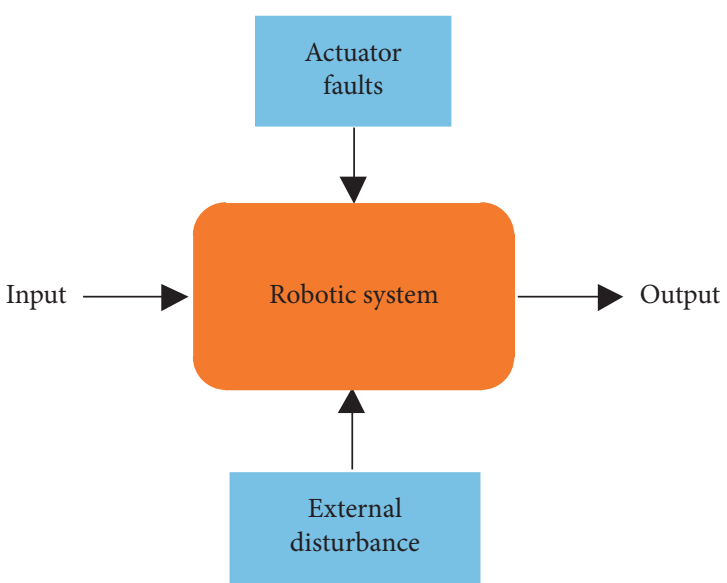

Figure 1: Robotic system with external disturbance and actuator faults.

Property 2. The matrix $\dot{M}(q)-2 C(q, \dot{q})$ is skew symmetric, i.e., $\xi^{T}(\dot{M}(q)-2 C(q, \dot{q})) \xi=0, \forall \xi \in R^{n \times 1}$.

2.2. Model of Actuator Faults. In a practical robotic system, the actuators may undergo fault due to aging, affecting the performance and even safety of the system. The mathematical model of actuator faults can be described as follows [13]:

$$
u_{f}(T)=u-u_{\text {nom }}
$$

where $u_{f}(T) \in R^{n \times 1}$ represents the actuator fault and $u_{\text {nom }} \in R^{n \times 1}$ represents the control torque from the nominal controller. Besides, $T=\left[\begin{array}{llll}T_{1} & T_{2} & \ldots & T_{n}\end{array}\right]^{T} \in R^{n \times 1}$ is the fault time-profile, where $T_{i}(i=1,2, \ldots, n)$ denotes the time at which the $i^{\text {th }}$ actuator undergoes fault. Generally, there are four types of actuator faults [23]:

(i) No fault: the controller is the nominal controller, i.e., $u=u_{\text {nom }}$ and $u_{f}(T)=0$.

(ii) Locked-in-place fault: the actuator fault is a constant, and the nominal controller is zero, i.e., $u=u_{f}(T), u_{\text {nom }}=0$, and $u_{f}(T)$ is a constant.

(iii) Loss of effectiveness fault: it means $u=D(t) u_{\text {nom }}+u_{f}(T) . u \in R^{n \times 1}$ is the actual control generated by the actuator. $D(t)=\operatorname{diag}\left[l_{1}(t), l_{2}(t), \ldots, l_{n}(t)\right]$ denotes the effectiveness of the actuator, where $0<l_{i}(t) \leq 1$ means that the $i^{\text {th }}$ actuator experiences a partial loss of effectiveness, and $u_{f}(T)=0, i=1,2, \ldots, n$.

(iv) Floating around trim fault: it can be accounted as $u=u_{\text {nom }}+u_{f}(T)$ and $u_{f}(T) \neq 0$.

\section{Composite Compensation Control of Robotic System}

For robotic system subject to external disturbance (1) and actuator faults (4), the structure of the proposed composite compensation control scheme is shown in Figure 2. First, a sliding mode controller is designed to suppress the external disturbance $\tau_{d}$. An adaptive law is employed to estimate the bound of the disturbance and obtain its estimation $\widehat{F}$. Then, a nonlinear observer is designed to directly estimate the state vector of the nonlinear function and obtain its estimation $\widehat{\alpha}(t)$ such that the actuator faults $u_{f}(T)$ can be indirectly estimated. A fault-tolerant controller is obtained based on the observer to compensate the actuator faults. Finally, the composite compensation controller $u_{\text {com }}$ is composed of the adaptive sliding mode controller $\tau_{\text {dcom }}$ and observer-based fault-tolerant controller $\tau_{\text {fcom }}$. Furthermore, the actual controller $u$ is obtained by combining the composite compensation controller $u_{\text {com }}$ and the nominal controller $u_{\text {nom }}$. In this way, the external disturbance and various actuator faults can be accurately compensated, and the real position $q$ of the robot can accurately track the desired position $q_{d}$.

3.1. Design of the Adaptive Sliding Mode Controller. Take $x_{1}=$ $q \in R^{n \times 1}$ and $x_{2}=\dot{q} \in R^{n \times 1}$ as the state variables of the system, and (1) can be directly rewritten into the state-space form as

$$
\left\{\begin{array}{l}
\dot{x}_{1}=x_{2}, \\
\dot{x}_{2}=M\left(x_{1}\right)^{-1}\left(u-\tau_{d}-C\left(x_{1}, x_{2}\right) x_{2}-G\left(x_{1}\right)\right) .
\end{array}\right.
$$

Define the sliding manifold as

$$
s=x_{2}-\phi,
$$

where $\phi$ is the state of the following nonlinear system (7):

$$
\begin{aligned}
\dot{\phi}= & M\left(x_{1}\right)^{-1}\left(k_{1} s+\widehat{F} \operatorname{sign}(s)+k_{2}|s|^{n_{1} / n_{2}} \cdot \operatorname{sign}\left(|s|^{n_{1} / n_{2}}\right)\right. \\
& \left.+u-G\left(x_{1}\right)-C\left(x_{1}, x_{2}\right) x_{2}+C\left(x_{1}, x_{2}\right) s\right),
\end{aligned}
$$

where $k_{1}>0$ and $k_{2}>0$ are positive constants and $n_{1}>0$ and $n_{2}>0$ are two odd integers satisfying $n_{1}<n_{2}$.

In order to suppress the external disturbance $\tau_{d}$ in (5), the sliding mode controller $\tau_{\mathrm{dcom}}$ can be designed as

$$
\tau_{\text {dcom }}=-k_{1} s-\widehat{F} \operatorname{sign}(s)-k_{2}|s|^{n_{1} / n_{2}} \cdot \operatorname{sign}\left(|s|^{n_{1} / n_{2}}\right) .
$$

As the bound of the external disturbance is usually unknown, an adaptive law is designed to estimate the bound F:

$$
\dot{\widehat{F}}=\beta s^{T} \operatorname{sign}(s),
$$

where $\widehat{F}$ is the estimation of $F, \beta>0$ is a positive constant, and sign is signum function.

3.2. Design of the Observer-Based Fault-Tolerant Controller. Let us introduce a new vector $M_{a}(q)=M(q) \dot{q}-\int_{0}^{t} e_{d}(l) \mathrm{d} l$, where $e_{d}=\tau_{\text {dcom }}-\tau_{d}$ is the disturbance compensation error. Then, from (1) and (4), we can get

$$
\dot{M}_{a}(q)=u_{\mathrm{nom}}+u_{f}(T)-\omega(q, \dot{q})-\tau_{\mathrm{dcom}},
$$

where $\omega(q, \dot{q})=C(q, \dot{q}) \dot{q}+G(q)-\dot{M}(q) \dot{q}$.

Now, define a new nominal controller $u_{\text {anom }}=u_{\text {nom }}+$ $\tau_{\mathrm{dcom}}$ and a new variable 


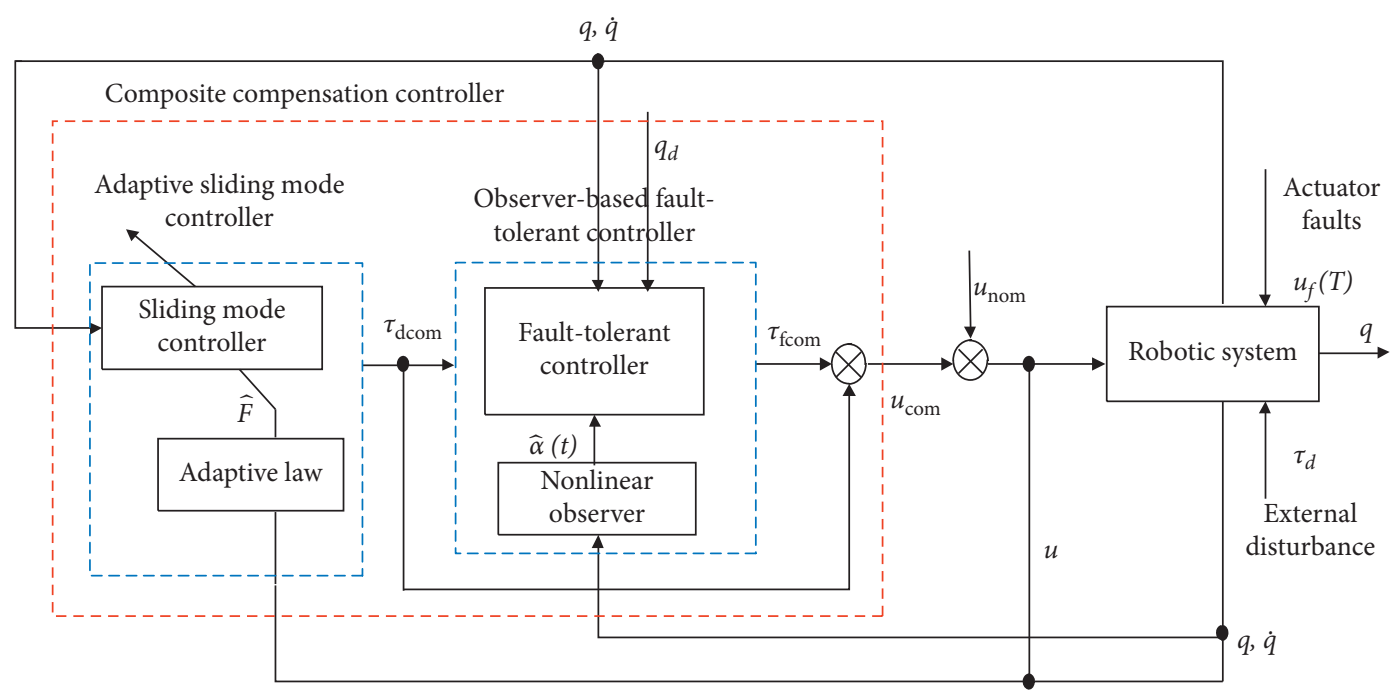

FIgURe 2: Structure of composite compensation control.

$\alpha(t)=k_{3} \int_{0}^{t}\left[u_{\mathrm{anom}}-\omega(q, \dot{q})-2 \tau_{\mathrm{dcom}}-\alpha(x)\right] \mathrm{d} x-k_{3} M_{a}(q)$,

where $k_{3}>0$ is a constant and $\alpha(t)$ is the state vector of the nonlinear function (11).

Differentiating (11) with respect to time and substituting (1) and (10) into it, we have

$$
\dot{\alpha}(t)=-k_{3} \alpha(t)-k_{3} u_{f}(T),
$$

where $u_{f}(T)$ can be regarded as the unknown input of the system (12).

As for the output of the system (12), we can take it as

$$
y=k_{4} \alpha(t)
$$

where $k_{4}>0$ is a positive constant.

Now, the fault $u_{f}(T)$ can be indirectly estimated by directly estimating the state $\alpha(t)$ of the system (12) through the following nonlinear observer

$$
\dot{\hat{\alpha}}(t)=-k_{3} \widehat{\alpha}(t)+\frac{1}{k_{4}} \dot{y}+k_{5} y+k_{6}|e|^{n_{1} / n_{2}},
$$

where $\widehat{\alpha}(t)$ is the estimation of $\alpha(t), e=\alpha-\widehat{\alpha}$ denotes the observation error of $\alpha(t)$, and $k_{5}=k_{3} / k_{4}$ and $k_{6}>0$ are observation gains.

Since $\alpha(t)$ can be estimated by the nonlinear observer (14), the fault-tolerant controller can be designed as

$$
\tau_{\mathrm{fcom}}=-\widehat{\alpha}(t)-\frac{1}{k_{3} k_{4}} \dot{y} .
$$

3.3. Design of the Composite Compensation Controller. With the adaptive sliding mode controller (8)-(9) and the observer-based fault-tolerant controller (14)-(15), the composite compensation controller $u_{\text {com }}$ can be designed as

$$
\begin{aligned}
u_{\text {com }}=\tau_{\text {dcom }}+\tau_{\text {fcom }}= & -k_{1} s-\widehat{F} \operatorname{sign}(s)-k_{2}|s|^{n_{1} / n_{2}} \\
& \cdot \operatorname{sign}\left(|s|^{n_{1} / n_{2}}\right)-\widehat{\alpha}(t)-\frac{1}{k_{3} k_{4}} \dot{y} .
\end{aligned}
$$

The composite compensation controller (16) can simultaneously compensate external disturbance and various types of actuator faults.

Theorem 1. Consider the nonlinear robotic system subject to external disturbance (1) and actuator faults (4). If it is controlled by the composite compensation controller (16), which is composed of the adaptive sliding mode controller (8)(9) and the observer-based fault-tolerant controller (14)-(15), then the disturbance compensation error and fault compensation error of the robotic system can converge to zero in finite time, i.e., $\lim _{t \longrightarrow t_{c}} e_{d}=\lim _{t \longrightarrow t_{c}}\left(\tau_{d c o m}-\tau_{d}\right)=0$ and $\lim _{t \rightarrow t_{c}} e_{f}=\lim _{t \longrightarrow t_{c}}\left(\tau_{\text {fcom }}-u_{f}(T)\right) \stackrel{t_{c}}{=} 0$.

Proof. Differentiating the observation error $e=\alpha-\widehat{\alpha}$ with respect to time and substituting (12)-(14) into it, we can obtain

$$
\begin{aligned}
\dot{e} & =\dot{\alpha}-\dot{\widehat{\alpha}} \\
& =-k_{3} \alpha-k_{3} u_{f}+k_{3} \widehat{\alpha}-\frac{1}{k_{4}} \dot{y}-k_{5} y-k_{6}|e|^{n_{1} / n_{2}} \\
& =-k_{3} e-k_{6}|e|^{n_{1} / n_{2}} .
\end{aligned}
$$

Define a Lyapunov function as

$$
V_{1}=\frac{1}{2} e^{T} e+\frac{1}{2} s^{T} M\left(x_{1}\right) s
$$

Differentiating (18) with respect to time and substituting (5)-(7) into it, we get 


$$
\begin{aligned}
\dot{V}_{1}= & e^{T} \dot{e}+\frac{1}{2} s^{T} \dot{M}\left(x_{1}\right) s+s^{T} M\left(x_{1}\right) \dot{s} \\
= & e^{T} \dot{e}+\frac{1}{2} s^{T} \dot{M}\left(x_{1}\right) s+s^{T} M\left(x_{1}\right)\left[M\left(x_{1}\right)^{-1}\right. \\
& \cdot\left(u-\tau_{d}-C\left(x_{1}, x_{2}\right) x_{2}\right. \\
& \left.-G\left(x_{1}\right)\right)-M\left(x_{1}\right)^{-1}\left(k_{1} s+F \operatorname{sign}(s)+k_{2}|s|^{n_{1} / n_{2}}\right. \\
& \left.\left.\cdot \operatorname{sign}\left(|s|^{n_{1} / n_{2}}\right)+u-G\left(x_{1}\right)-C\left(x_{1}, x_{2}\right) x_{2}+C\left(x_{1}, x_{2}\right) s\right)\right] \\
= & e^{T} \dot{e}+\frac{1}{2} s^{T} \dot{M}\left(x_{1}\right) s-s^{T} \tau_{d}-k_{1} s^{T} s-F s^{T} \operatorname{sign}(s) \\
& -s^{T} C\left(x_{1}, x_{2}\right) s-k_{2} s^{T} \cdot\left(|s|^{n_{1} / n_{2}} \cdot \operatorname{sign}\left(|s|^{n_{1} / n_{2}}\right)\right) .
\end{aligned}
$$
get

Substituting (17) into (19) and using Property 2, we can

$$
\begin{aligned}
\dot{V}_{1}= & e^{T}\left(-k_{3} e-k_{6}|e|^{n_{1} / n_{2}}\right)+\frac{1}{2} s^{T} \dot{M}\left(x_{1}\right) s-s^{T} \tau_{d}-k_{1} s^{T} s \\
& -F s^{T} \operatorname{sign}(s) \\
& -s^{T} C\left(x_{1}, x_{2}\right) s-k_{2} s^{T} \cdot\left(|s|^{n_{1} / n_{2}} \cdot \operatorname{sign}\left(|s|^{n_{1} / n_{2}}\right)\right) \\
= & -k_{3} e^{T} e-k_{6} e^{T}|e|^{n_{1} / n_{2}}-s^{T} \tau_{d}-k_{1} s^{T} s-F s^{T} \operatorname{sign}(s) \\
& -k_{2} s^{T} \cdot\left(|s|^{n_{1} / n_{2}} \cdot \operatorname{sign}\left(|s|^{n_{1} / n_{2}}\right)\right) .
\end{aligned}
$$

According to Property 1 and Property 2, (20) becomes

$$
\begin{aligned}
\dot{V}_{1} \leq & -2 k_{3}\left(\frac{1}{2} e^{T} e\right)-2^{\left(n_{1}+n_{2}\right) / 2 n_{2}} k_{6}\left(\frac{1}{2} e^{T} e\right)^{\left(n_{1}+n_{2}\right) / 2 n_{2}} \\
& +\|s\|\left\|\tau_{d}\right\|-\frac{2 k_{1}}{\lambda_{1}}\left(\frac{1}{2} s^{T} M\left(x_{1}\right) s\right) \\
& -F\|s\|-k_{2}\left(\frac{2}{\lambda_{1}}\right)^{\left(n_{1}+n_{2}\right) / 2 n_{2}}\left(\frac{1}{2} s^{T} M\left(x_{1}\right) s\right)^{\left(n_{1}+n_{2}\right) / 2 n_{2}} \\
\leq & -2 k_{3}\left(\frac{1}{2} e^{T} e\right)-2^{\left(n_{1}+n_{2}\right) / 2 n_{2}} k_{6}\left(\frac{1}{2} e^{T} e\right)^{\left(n_{1}+n_{2}\right) / 2 n_{2}} \\
& -\frac{2 k_{1}}{\lambda_{1}}\left(\frac{1}{2} s^{T} M\left(x_{1}\right) s\right)^{\left(n_{1}+n_{2}\right) / 2 n_{2}}\left(\frac{1}{2} s^{T} M\left(x_{1}\right) s\right)^{\left(n_{1}+n_{2}\right) / 2 n_{2}} . \\
& -k_{2}\left(\frac{2}{\lambda_{1}}\right)^{\left(n^{T}\right.} .
\end{aligned}
$$

Now, let $k_{7}=2 k_{3}, k_{8}=2 k_{1} / \lambda_{1}, k_{9}=2^{\left(n_{1}+n_{2}\right) / 2 n_{2}} k_{6}$, and $k_{10}=\left(2 / \lambda_{2}\right)^{\left(n_{1}+n_{2}\right) / 2 n_{2}} k_{2}$, and we can further obtain

$$
\begin{aligned}
\dot{V}_{1} \leq & -k_{7}\left(\frac{1}{2} e^{T} e\right)-k_{8}\left(\frac{1}{2} s^{T} M\left(x_{1}\right) s\right)-k_{9}\left(\frac{1}{2} e^{T} e\right)^{\left(n_{1}+n_{2}\right) / 2 n_{2}} \\
& -k_{10}\left(\frac{1}{2} s^{T} M\left(x_{1}\right) s\right)^{\left(n_{1}+n_{2}\right) / 2 n_{2}} \\
\leq & -c_{1}\left(\frac{1}{2} e^{T} e+\frac{1}{2} s^{T} M\left(x_{1}\right) s\right) \\
& -c_{2}\left(\frac{1}{2} e^{T} e+\frac{1}{2} s^{T} M\left(x_{1}\right) s\right)^{\left(n_{1}+n_{2}\right) / 2 n_{2}} \\
= & -c_{1} V_{1}-c_{2} V_{1}^{\left(n_{1}+n_{2}\right) / 2 n_{2}}
\end{aligned}
$$

where $\quad c_{1}=\min \left\{k_{7}, k_{8}\right\} \quad$ and $\quad c_{2}=\min \left\{k_{9}, k_{10}\right\} \quad$ and $0<\left(\left(n_{1}+n_{2}\right) / 2 n_{2}\right)<1$. Solving (22) leads to $V_{1}(t) \equiv 0$ for all $t \geq t_{c}$. Therefore, from (22), it is easy to show that $\dot{V}_{1}(t) \leq 0$ and the finite time $t_{c}$ can be obtained as

$$
t_{c} \leq \frac{2 n_{2}}{c_{1}\left(n_{2}-n_{1}\right)} \ln \frac{c_{1} V_{1}^{\left(n_{2}-n_{1}\right) / 2 n_{2}}(0)+c_{2}}{c_{2}} .
$$

Now, define another Lyapunov function as

$$
V_{2}=V_{1}+\frac{1}{2 \beta} \widetilde{F}^{2}
$$

where $\widetilde{F}=F-\widehat{F}$ is the estimation error of $F$.

Differentiating (24) with respect to time and substituting (5)-(7) into it yield

$$
\begin{aligned}
\dot{V}_{2}= & \dot{V}_{1}-\frac{1}{\beta}(F-\widehat{F}) \dot{\widehat{F}} \\
= & e^{T} \dot{e}+\frac{1}{2} s^{T} \dot{M}\left(x_{1}\right) s+s^{T} M\left(x_{1}\right) \dot{s}-\frac{1}{\beta}(F-\widehat{F}) \dot{\widehat{F}} \\
= & e^{T} \dot{e}+\frac{1}{2} s^{T} \dot{M}\left(x_{1}\right) s+s^{T} M\left(x_{1}\right)\left[M\left(x_{1}\right)^{-1}\right. \\
& \cdot\left(u-\tau_{d}-C\left(x_{1}, x_{2}\right) x_{2}\right. \\
& \left.-G\left(x_{1}\right)\right)-M\left(x_{1}\right)^{-1}\left(k_{1} s+\widehat{F} \operatorname{sign}(s)+k_{2}|s|^{n_{1} / n_{2}}\right. \\
& \cdot \operatorname{sign}\left(|s|^{n_{1} / n_{2}}\right) \\
& \left.\left.+u-G\left(x_{1}\right)-C\left(x_{1}, x_{2}\right) x_{2}+C\left(x_{1}, x_{2}\right) s\right)\right]-\frac{1}{\beta}(F-\widehat{F}) \dot{\widehat{F}} \\
= & e^{T} \dot{e}+\frac{1}{2} s^{T} \dot{M}\left(x_{1}\right) s-s^{T} \tau_{d}-k_{1} s^{T} s-\widehat{F} s^{T} \operatorname{sign}(s) \\
& -s^{T} C\left(x_{1}, x_{2}\right) s \\
& \left.-k_{2} s^{T} \cdot\left(|s|^{n_{1} / n_{2}} \cdot \operatorname{sign}\left(|s|^{n_{1} / n_{2}}\right)\right)-\frac{1}{\beta}(F-\widehat{F}) \dot{\widehat{F}}\right) . \\
&
\end{aligned}
$$

Substituting (9) and (17) into (25) and using Property 2 give us 


$$
\begin{aligned}
\dot{V}_{2}= & e^{T}\left(-k_{3} e-k_{6}|e|^{n_{1} / n_{2}}\right)+\frac{1}{2} s^{T} \dot{M}\left(x_{1}\right) s-s^{T} \tau_{d}-k_{1} s^{T} s \\
& -\widehat{F} s^{T} \operatorname{sign}(s)-k_{2} s^{T} \cdot\left(|s|^{n_{1} / n_{2}} \cdot \operatorname{sign}\left(|s|^{n_{1} / n_{2}}\right)\right) \\
& -s^{T} C\left(x_{1}, x_{2}\right) s-\frac{1}{\beta}(F-\widehat{F}) \dot{\widehat{F}} \\
= & -k_{3} e^{T} e-k_{6} e^{T}|e|^{n_{1} / n_{2}}-s^{T} \tau_{d}-k_{1} s^{T} s-\widehat{F} s^{T} \operatorname{sign}(s) \\
& -k_{2} s^{T} \cdot\left(|s|^{n_{1} / n_{2}} \cdot \operatorname{sign}\left(|s|^{n_{1} / n_{2}}\right)\right)-F s^{T} \operatorname{sign}(s) \\
& +\widehat{F}^{T} \operatorname{sign}(s) .
\end{aligned}
$$

Using Property 1 and Property 2, we have

$$
\begin{aligned}
\dot{V}_{2} \leq & -k_{3}\|e\|^{2}-k_{6} e^{T}|e|^{n_{1} / n_{2}}+\|s\|\left\|\tau_{d}\right\|-k_{1}\|s\|^{2} \\
& -k_{2} s^{T}|s|^{n_{1} / n_{2}}-F\|s\| \\
\leq & -2 k_{3}\left(\frac{1}{2} e^{T} e\right)-2^{\left(n_{1}+n_{2}\right) / 2 n_{2}} k_{6}\left(\frac{1}{2} e^{T} e\right)^{\left(n_{1}+n_{2}\right) / 2 n_{2}} \\
& -\frac{2 k_{1}}{\lambda_{2}}\left(\frac{1}{2} s^{T} M\left(x_{1}\right) s\right) \\
& -k_{2}\left(\frac{2}{\lambda_{2}}\right)^{\left(n_{1}+n_{2}\right) / 2 n_{2}}\left(\frac{1}{2} s^{T} M\left(x_{1}\right) s\right)^{\left(n_{1}+n_{2}\right) / 2 n_{2}} \\
= & -k_{7}\left(\frac{1}{2} e^{T} e\right)-k_{8}\left(\frac{1}{2} s^{T} M\left(x_{1}\right) s\right)-k_{9}\left(\frac{1}{2} e^{T} e\right)^{\left(n_{1}+n_{2}\right) / 2 n_{2}} \\
& -k_{10}\left(\frac{1}{2} s^{T} M\left(x_{1}\right) s\right)^{\left(n_{1}+n_{2}\right) / 2 n_{2}} \\
\leq & -c_{1}\left(\frac{1}{2} e^{T} e+\frac{1}{2} s^{T} M\left(x_{1}\right) s\right)-c_{2}\left(\frac{1}{2} e^{T} e+\frac{1}{2} s^{T} M\left(x_{1}\right) s\right)^{\left(n_{1}+n_{2}\right) / 2 n_{2}} \\
= & -c_{1} V_{1}-c_{2} V_{1}^{\left(n_{1}+n_{2}\right) / 2 n_{2}} .
\end{aligned}
$$

Solving (27) leads to $V_{1}(t) \equiv 0$ for all $t \geq t_{c}$. Therefore, from (27), we can obtain $\dot{V}_{2}(t) \leq 0$.

From (5) and (8), the disturbance compensation error can be derived as

$$
\begin{aligned}
e_{d}= & \tau_{\text {dcom }}-\tau_{d} \\
= & -k_{1} s-\widehat{F} \operatorname{sign}(s)-k_{2}|s|^{n_{1} / n_{2}} \cdot \operatorname{sign}\left(|s|^{n_{1} / n_{2}}\right)-u \\
& +C\left(x_{1}, x_{2}\right) x_{2}+G\left(x_{1}\right)+M\left(x_{1}\right) \dot{x}_{2} .
\end{aligned}
$$

Substituting (6) and (7) into (28), we can get

$$
\begin{aligned}
e_{d}= & -k_{1} s-\widehat{F} \operatorname{sign}(s)-k_{2}|s|^{n_{1} / n_{2}} \cdot \operatorname{sign}\left(|s|^{n_{1} / n_{2}}\right) \\
& -u+M\left(x_{1}\right) \dot{s}+M\left(x_{1}\right) \dot{\phi}+C\left(x_{1}, x_{2}\right) x_{2}+G\left(x_{1}\right) \\
= & M\left(x_{1}\right) \dot{s}+C\left(x_{1}, x_{2}\right) s .
\end{aligned}
$$

From (12), (13), and (15), the fault compensation error can be derived as

$$
\begin{aligned}
e_{f} & =\tau_{\mathrm{fcom}}-u_{f} \\
& =-\widehat{\alpha}(t)-\frac{1}{k_{3} k_{4}} \dot{y}-\left(-\alpha(t)-\frac{1}{k_{3}} \dot{\alpha}(t)\right) \\
& =-\widehat{\alpha}(t)+\alpha(t) \\
& =-e .
\end{aligned}
$$

Solving (27) leads to $V_{2}(t) \equiv 0$ for all $t \geq t_{c}$. Then, according to (18), we have $e(t)=0$ and $s(t)=0$ for all $t \geq t_{c}$. Thus, we can further have $\dot{s}(t)=0$. Therefore, from (29) and (30), we can get $e_{d}=0$ and $e_{f}=0$ for all $t \geq t_{c}$. This indicates that $e_{d}$ and $e_{f}$ can converge to zero in finite time $t_{c}$, i.e., $\lim _{t \longrightarrow t_{c}} e_{d}=\lim _{t \longrightarrow t_{c}}\left(\tau_{\mathrm{dcom}}-\tau_{d}\right)=0$ and $\lim _{t \longrightarrow t_{c}} e_{f}=$ $\lim _{t \longrightarrow t_{c}}\left(\tau_{\text {fcom }}-u_{f}(T)\right)=0$. This concludes the proof of Theorem 1 .

Remark 1. It can be seen from the nonlinear observer (14) and the proof of Theorem 1 that the nominal controller $u_{\text {nom }}$ can be cancelled in the composite compensation controller (16). This indicates that the proposed composite compensation control scheme does not depend on the specific nominal control law.

Remark 2. In the literature [18-20], disturbance and fault are treated as centralized uncertainty. Different from them, the designed composite compensation controller (16) consists the terms regarding disturbance as well as actuator faults. Thus, the respective characteristic of disturbance and actuator faults can better be reflected.

\section{Simulations}

Simulations are conducted on a 2-DOF robot manipulator, as shown in Figure 3. The dynamics of the robot is

$$
\begin{aligned}
M(q) & =\left[\begin{array}{cc}
p_{1}+p_{2}+2 p_{3} \cos q_{2} & p_{2}+p_{3} \cos q_{2} \\
p_{2}+p_{3} \cos q_{2} & p_{2}
\end{array}\right], \\
C(q, \dot{q}) & =\left[\begin{array}{cc}
-p_{3} \dot{q}_{2} \sin q_{2} & -p_{3}\left(\dot{q}_{1}+\dot{q}_{2}\right) \sin q_{2} \\
p_{3} \dot{q}_{1} \sin q_{2} & 0
\end{array}\right], \\
G(q) & =\left[\begin{array}{c}
p_{4} g \cos q_{1}+p_{5} g \cos \left(q_{1}+q_{2}\right) \\
p_{5} g \cos \left(q_{1}+q_{2}\right)
\end{array}\right],
\end{aligned}
$$

where $q=\left[\begin{array}{ll}q_{1} & q_{2}\end{array}\right]^{T}$ and $q_{1}$ and $q_{2}$ represent the position of the first joint and the second joint, respectively. Besides,

$$
\left[\begin{array}{c}
p_{1} \\
p_{2} \\
p_{3} \\
p_{4} \\
p_{5}
\end{array}\right]=\left[\begin{array}{c}
m_{1} h_{1}^{2}+m_{2} l_{1}^{2}+J_{1} \\
m_{2} h_{2}^{2}+J_{2} \\
m_{2} l_{1} h_{2} \\
m_{1} h_{1}+m_{2} l_{1} \\
m_{2} h_{2}
\end{array}\right],
$$

where $m_{1}$ and $m_{2}$ are the mass of the link, $l_{1}$ and $l_{2}$ are the length of the link, $h_{1}$ and $h_{2}$ are the distance to the center of the mass, $J_{1}$ and $J_{2}$ are the moment of inertia, and $g$ is the 


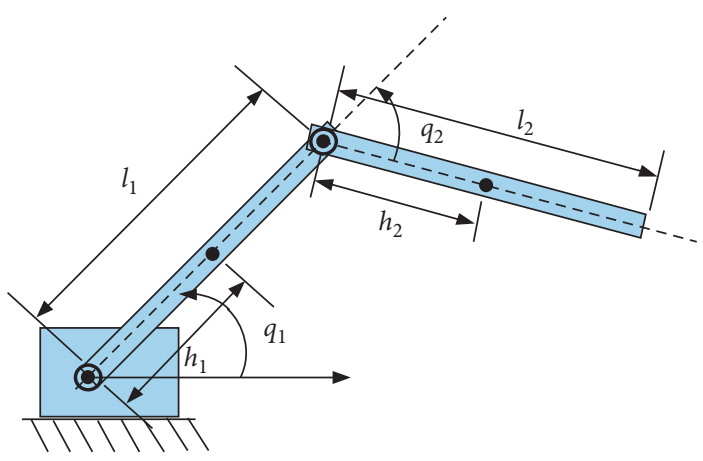

FIGURE 3: 2-DOF robot manipulator.

gravity coefficient. In the simulations, $m_{1}=m_{2}=1$, $l_{1}=l_{2}=1, h_{1}=h_{2}=0.5$, and $J_{1}=J_{2}=0.08$.

The conventional PD controller [24] which is widely applied in practice is taken as the nominal controller $u_{\text {nom }}$ :

$$
u_{\mathrm{nom}}=M(q)\left[\ddot{q}_{d}+k_{d} \dot{e}_{p}+k_{p} e_{p}\right]+C(q, \dot{q})+G(q),
$$

where $e_{p}=q_{d}-q$ represents the position tracking error of the robot.

The initial value of the robot is $q(0)=\left[\begin{array}{ll}0.05 & 0.1\end{array}\right]^{T}$. The desired position of the robot is $q_{d}=\left[\begin{array}{ll}q_{d 1} & q_{d 2}\end{array}\right]^{T}$, where

$$
\left\{\begin{array}{l}
q_{d 1}=0.05 \sin (4 t-0.5 \pi), \\
q_{d 2}=0.06 \sin (4 t-0.5 \pi)
\end{array}\right.
$$

The external disturbance in the robotic system is

$$
\tau_{d}=\left[\begin{array}{ll}
0.2 \cos (2 t) & 0.4 \cos (2 t)
\end{array}\right]^{T} .
$$

For joint 1 of the robot, during $2 \mathrm{sec}-3 \mathrm{sec}$ and $8 \mathrm{sec}-9 \mathrm{sec}$, the actuator fault is constant deviation fault. During $4 \mathrm{sec}-7 \mathrm{sec}$, the actuator fault is time-varying fault. For the rest of the time, the actuator is no fault.

For joint 2 of the robot, during $0 \mathrm{sec}-5 \mathrm{sec}$, the actuator is no fault. After $5 \mathrm{sec}$, the actuator fault is loss of effectiveness fault; i.e., the actuator losses $20 \%$ of the effectiveness.

Specifically, the actuator fault for joint 1 and joint 2 is as follows:

$$
\begin{aligned}
& u_{f_{1}}\left(T_{1}\right)=\left\{\begin{array}{ll}
-0.8, & 2 \leq t \leq 3, \\
-0.6 \sin (4 t), & 4 \leq t \leq 7, \\
-0.5, & 8 \leq t \leq 9, \\
0, & \text { elsewhere }
\end{array},\right. \\
& u_{f_{2}}\left(T_{2}\right)= \begin{cases}-0.2 u_{\text {nom }}, & t \geq 5, \\
0, & \text { elsewhere. }\end{cases}
\end{aligned}
$$

In the simulations, the performances of the conventional fault reconstruction scheme [13] and the proposed composite compensation control scheme are compared. The parameters of the conventional fault reconstruction scheme [13] are chosen as $k_{d}=70, k_{p}=50, k_{1}=0.001$, $k_{2}=25, k_{3}=75, k_{4}=145, n_{1}=101$, and $n_{2}=103$. The parameters of the proposed composite compensation controller are chosen as $k_{p}=800, k_{d}=500, k_{1}=1165$, $k_{2}=680, \quad k_{3}=800, \quad k_{4}=50, \quad k_{5}=k_{3} / k_{4}=16, \quad k_{6}=0.5$, $n_{1}=87, n_{2}=103$, and $\beta=0.5$. The simulation results are shown in Figures $4-9$.

The effect of the external disturbance compensation with the composite compensation controller is shown in Figures 4 and 5. It can be seen that the proposed composite compensation controller can successfully compensate the disturbance, and the disturbance compensation error can quickly converge within a short time. Since the conventional fault reconstruction scheme cannot compensate the external disturbance, the effect of the external disturbance compensation with the conventional fault reconstruction scheme is not shown.

Figures 6(a) and 6(b) show that both the conventional fault reconstruction scheme and the proposed composite compensation controller can compensate various types of actuator faults. However, it can be seen from Figures 7(a) and $7(\mathrm{~b})$ that when the proposed controller is employed, the fault compensation accuracy is higher and the fault error convergence rate is faster.

Figure 8(a) shows that, with the conventional fault reconstruction scheme, the real position trajectory of the robot cannot track the desired position trajectory well. Comparatively, Figure 8(b) shows that, with the proposed composite compensation controller, the robot can track the desired position in a satisfactory way within a short time. As shown in Figure 9(a), when the fault reconstruction scheme is used, there exists obvious position tracking error, and the error convergence rate is slow. Comparatively, when the proposed controller is employed, the position tracking error is ideal, and the error convergence rate is faster in Figure 9(b). The reason is that the proposed composite compensation controller can not only deal with actuator faults but also external disturbance in the system.

To further demonstrate the superiority of the proposed composite compensation control scheme, several performance indicators are compared in quantitative in Tables 1-3. The indicator $t_{d_{i} s}$ denotes the adjustment time of disturbance compensation, and $\left|e_{d_{i}}\right|$ represents the disturbance compensation error. $t_{f_{i} s}$ denotes the adjustment time of fault compensation, and $\left|e_{f_{i}}\right|$ represents the fault compensation error. $t_{p_{i} s}$ denotes the adjustment time of position tracking, 


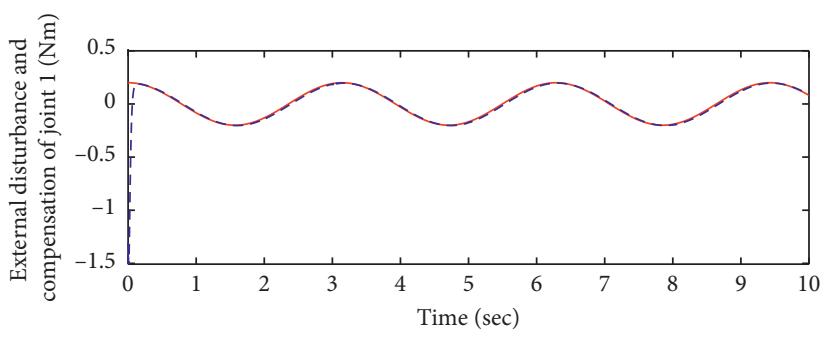

- Real disturbance of joint 1

- - - Disturbance compensation of joint 1

(a)

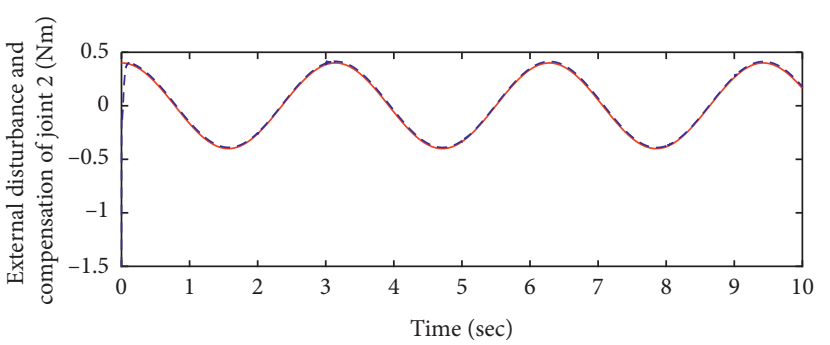

- Real disturbance of joint 2

- - - Disturbance compensation of joint 2

(b)

Figure 4: External disturbance and compensation of joint 1 and joint 2 (composite compensation controller).

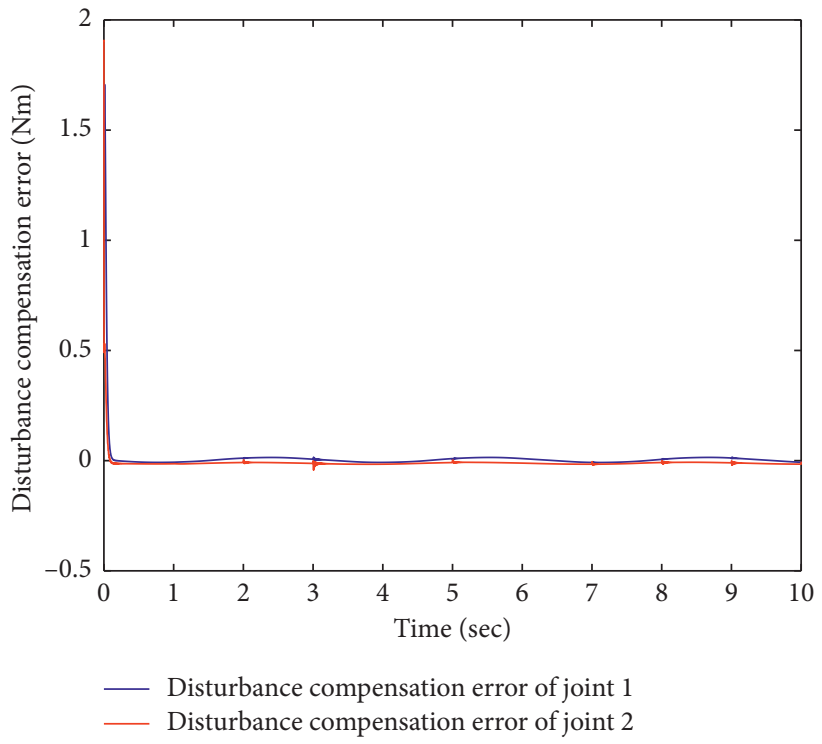

FIGURE 5: Disturbance compensation error of joint 1 and joint 2 (composite compensation controller).
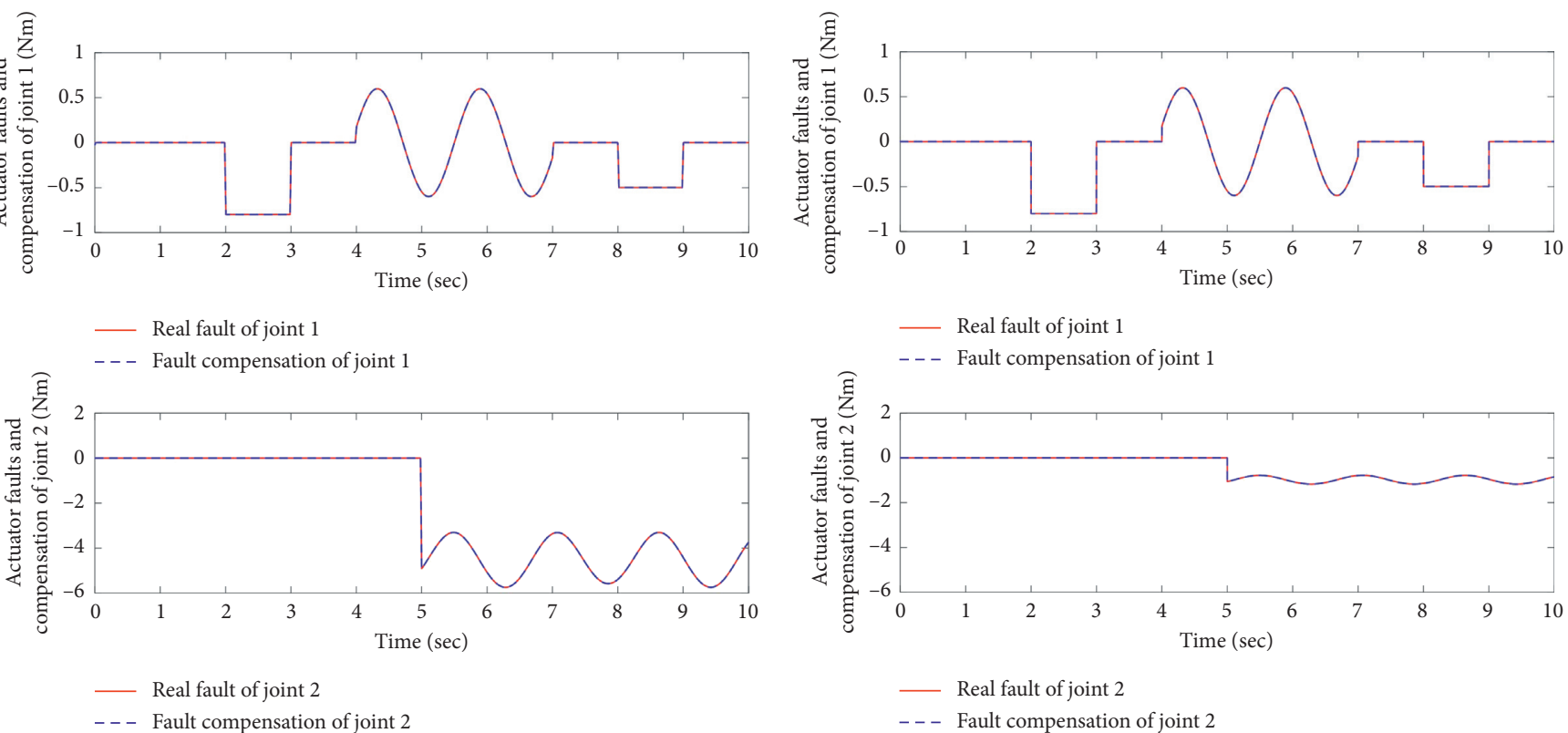

(a)

(b)

FiguRE 6: Actuator faults and compensation of joint 1 and joint 2: (a) fault reconstruction scheme [13]; (b) composite compensation controller. 


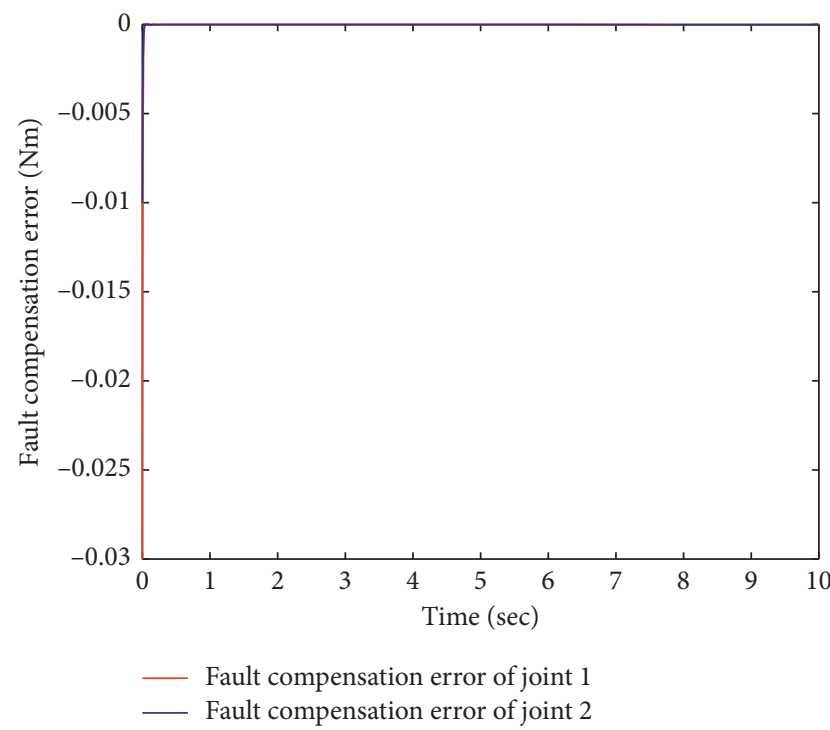

(a)

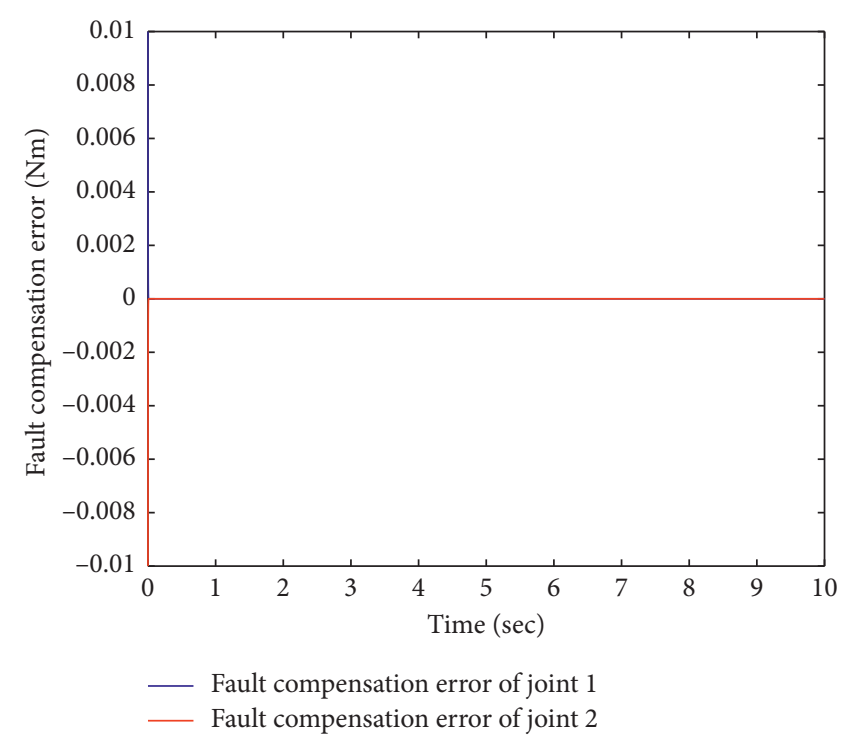

(b)

Figure 7: Fault compensation error of joint 1 and joint 2: (a) fault reconstruction scheme [13]; (b) composite compensation controller.

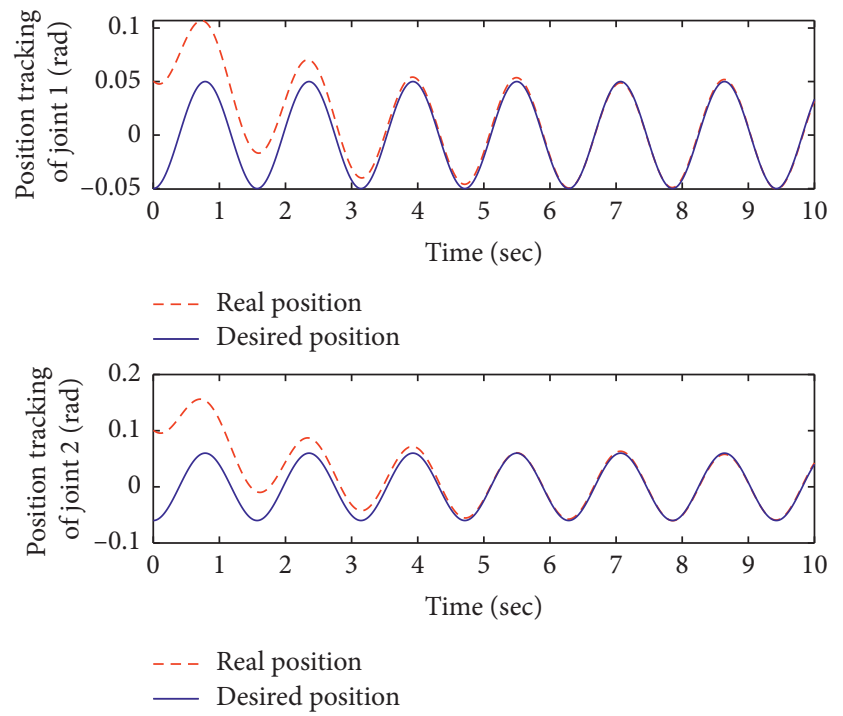

(a)
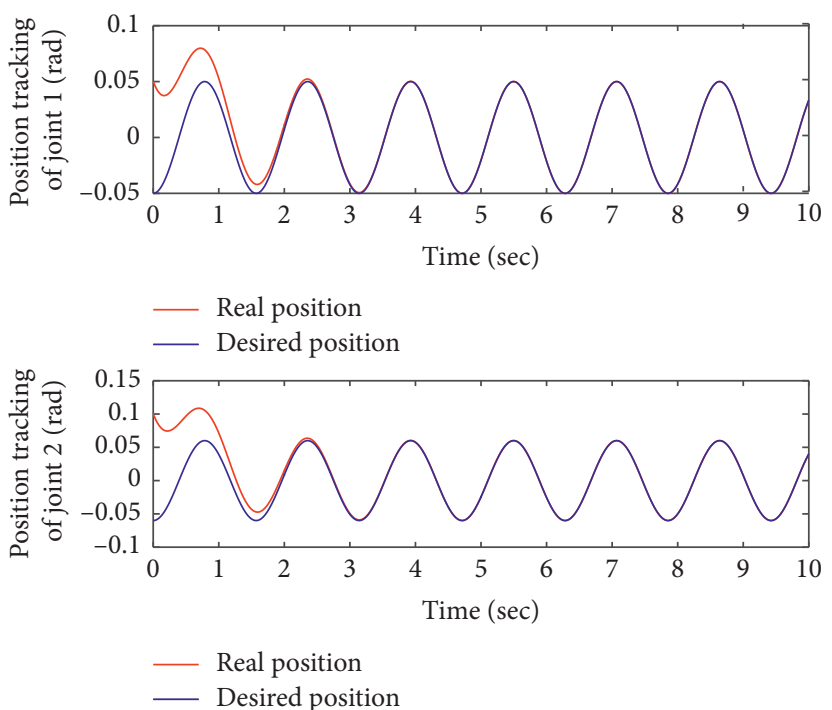

(b)

Figure 8: Position tracking of joint 1 and joint 2: (a) fault reconstruction scheme [13]; (b) composite compensation controller.

and $\left|e_{p_{i}}\right|_{\max }$ represents the position tracking error, where $i=$ 1,2 represent joint 1 and joint 2 of the robot, respectively.

Table 1 indicates that, with the proposed composite compensation controller, the disturbance compensation error of joint 1 and joint 2 can rapidly converge in $0.1191 \mathrm{sec}$ and $0.0833 \mathrm{sec}$, respectively. Nevertheless, the conventional fault reconstruction scheme cannot compensate disturbance.

Table 2 shows that, with the proposed composite compensation controller, the adjustment time of fault compensation is shorter and the absolute value of the fault compensation error is smaller. In other words, when the proposed controller is employed, the fault compensation accuracy is higher and the fault error convergence rate is faster.

Table 3 shows that, with the proposed composite compensation controller, the adjustment time of position tracking for both joint 1 and joint 2 is shorter, and the absolute value of the position tracking error is smaller. In other words, when the proposed controller is employed, the robot can track the desired position trajectory more accurately and quickly. 


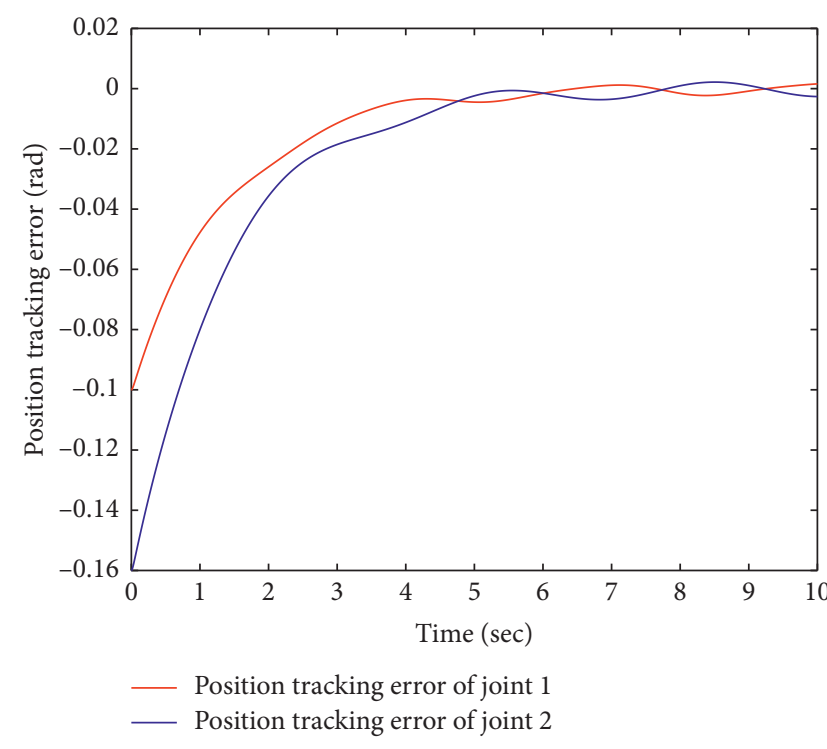

(a)

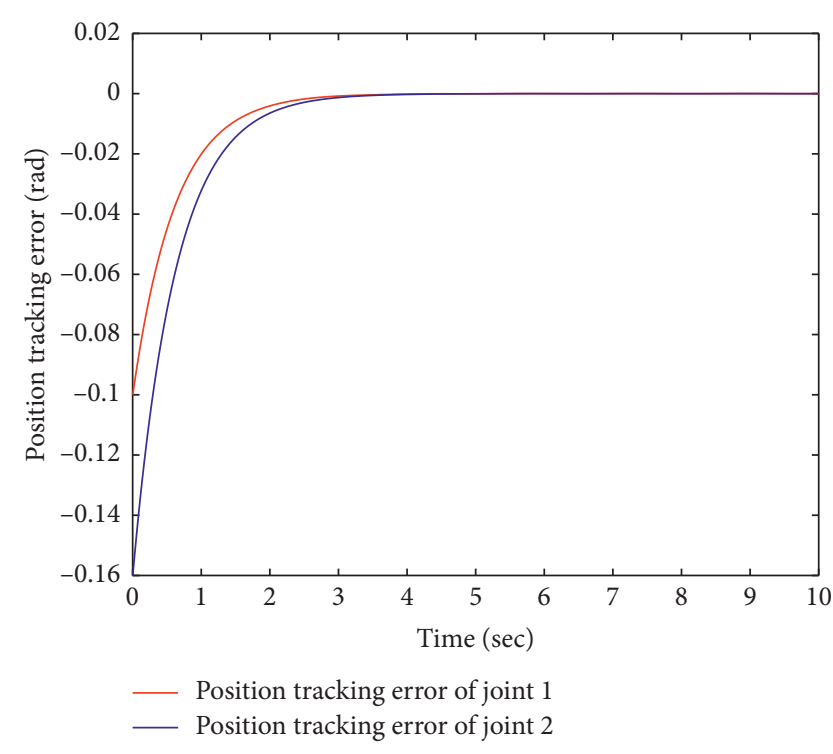

(b)

FIGURE 9: Position tracking error of joint 1 and joint 2: (a) fault reconstruction scheme [13]; (b) composite compensation controller.

TABle 1: Quantitative comparison of external disturbance compensation.

\begin{tabular}{lcccc}
\hline & \multicolumn{2}{c}{ Composite compensation controller } & \multicolumn{2}{c}{ Fault reconstruction scheme } \\
& $t_{d_{i} s}( \pm 2 \%)(\mathrm{sec})$ & $\left|e_{d_{i}}\right|_{\max }(\mathrm{Nm})$ & $t_{d_{i} s}( \pm 2 \%)(\mathrm{sec})$ & None \\
\hline Joint 1 & 0.1191 & $1.147 \times 10^{-2}$ & None & \\
Joint 2 & 0.0833 & $1.53 \times 10^{-2}$ & None & None \\
\hline
\end{tabular}

TABLE 2: Quantitative comparison of actuator fault compensation.

\begin{tabular}{lccrr}
\hline & \multicolumn{2}{c}{ Composite compensation controller } & \multicolumn{2}{c}{ Fault reconstruction scheme } \\
& $t_{f_{i} s}( \pm 5 \%)(\mathrm{sec})$ & $\left|e_{f_{i}}\right|_{\max }(\mathrm{Nm})$ & $t_{f_{i} s}( \pm 5 \%)(\mathrm{sec})$ & 0.044 \\
\hline Joint 1 & 0.006 & $1.11 \times 10^{-16}$ & 0.044 & $1.89 \times 10^{-5}$ \\
Joint 2 & 0.006 & $8.88 \times 10^{-15}$ & $0.89 \times 10^{-5}$ \\
\hline
\end{tabular}

TABLE 3: Quantitative comparison of position tracking.

\begin{tabular}{lcccc}
\hline & \multicolumn{2}{c}{ Composite compensation controller } & \multicolumn{2}{c}{ Fault reconstruction scheme } \\
& $t_{p_{i} s}( \pm 3 \%)(\mathrm{sec})$ & $\left|e_{p_{i}}\right|_{\max }(\mathrm{rad})$ & $t_{p_{i} s}( \pm 3 \%)(\mathrm{sec})$ & 5.777 \\
\hline Joint 1 & 2.435 & $3.32 \times 10^{-5}$ & 4.77 & $2.49 \times 10^{-3}$ \\
Joint 2 & 2.734 & $3.65 \times 10^{-6}$ & 4.834 & $3.57 \times 10^{-3}$ \\
\hline
\end{tabular}

\section{Conclusions}

For a robotic system subject to simultaneous external disturbance and various actuator faults, a composite compensation control scheme based on adaptive sliding mode controller and observer-based fault-tolerant controller is proposed. Compared to the conventional fault reconstruction scheme, the proposed scheme can compensate not only external disturbance but also various actuator faults. The fault compensation accuracy is higher, and the fault error convergence rate is faster. Moreover, the robot can track the desired position trajectory more accurately and quickly. Experimental verification of the proposed control in this paper is quite necessary and remains as our work in the next step. Besides, the extension of the proposed control to online estimates the fault information for a nonlinear robotic system using a fault diagnosis approach remains as our future research.

\section{Data Availability}

The data that support our manuscript conclusions are some open access articles that have been properly cited, and the 
readers can easily obtain these articles to verify the conclusions.

\section{Conflicts of Interest}

The authors declare that they have no conflicts of interest regarding the publication of this paper.

\section{Acknowledgments}

This research was supported by the National Natural Science Foundation of China (Grant nos. 61973257, 61973331, and 61875166) and Sichuan Youth Science and Technology Foundation (Grant 2017JQ0022).

\section{References}

[1] Y. Guo, "Globally robust stability analysis for stochastic cohen-grossberg neural networks with impulse control and time-varying delays," Ukrainian Mathematical Journal, vol. 69 , no. 8, pp. 1220-1233, 2017.

[2] W. Liu, J. Cui, and J. Xin, "A block-centered finite difference method for an unsteady asymptotic coupled model in fractured media aquifer system," Journal of Computational and Applied Mathematics, vol. 337, pp. 319-340, 2018.

[3] L. Gao, D. Wang, and G. Wang, "Further results on exponential stability for impulsive switched nonlinear time-delay systems with delayed impulse effects," Applied Mathematics and Computation, vol. 268, pp. 186-200, 2015.

[4] V. Utkin, Sliding Modes on Control and Optimization, Springer-Verlag, Berlin, Germany, 1992.

[5] W.-H. Chen, "Disturbance observer based control for nonlinear systems," IEEE/ASME Transactions on Mechatronics, vol. 9, no. 4, pp. 706-710, 2004.

[6] Z. Pu, R. Yuan, J. Yi, and X. Tan, "A class of adaptive extended state observers for nonlinear disturbed systems," IEEE Transactions on Industrial Electronics, vol. 62, no. 9, pp. 5858-5869, 2015.

[7] H. Wang, Y. Pan, S. Li, and H. Yu, "Robust sliding mode control for robots driven by compliant actuators," IEEE Transactions on Control Systems Technology, vol. 27, no. 3, pp. 1259-1266, 2019.

[8] R. Cui, L. Chen, C. Yang, and M. Chen, "Extended state observer-based integral sliding mode control for an underwater robot with unknown disturbances and uncertain nonlinearities," IEEE Transactions on Industrial Electronics, vol. 64, no. 8, pp. 6785-6795, 2017.

[9] W. Yuan and G. Gao, "Sliding mode control of the automobile electro-coating conveying mechanism with a nonlinear disturbance observer," Advances in Mechanical Engineering, vol. 10, no. 9, pp. 1-9, 2018.

[10] M. Li and Y. Chen, "Robust adaptive sliding mode control for switched networked control systems with disturbance and faults," IEEE Transactions on Industrial Informatics, vol. 15, no. 1, pp. 193-204, 2019.

[11] Q. Meng, T. Zhang, X. Gao, and J.-y. Song, “Adaptive sliding mode fault-tolerant control of the uncertain stewart platform based on offline multibody dynamics," IEEE/ASME Transactions on Mechatronics, vol. 19, no. 3, pp. 882-894, 2014.

[12] M. S. Mahmoud, A. M. Memon, and P. Shi, "Observer-based fault-tolerant control for a class of nonlinear networked control systems," International Journal of Control, vol. 87, no. 8, pp. 1707-1715, 2014.
[13] B. Xiao and S. Yin, "An intelligent actuator fault reconstruction scheme for robotic manipulators," IEEE Transactions on Cybernetics, vol. 48, no. 2, pp. 639-647, 2018.

[14] J. Zhao, S. Jiang, F. Xie, Z. He, and J. Fu, "A novel nonlinear fault tolerant control for manipulator under actuator fault," Mathematical Problems in Engineering, vol. 2018, Article ID 5198615, 11 pages, 2018.

[15] B. Xiao, S. Yin, and H. Gao, "Reconfigurable tolerant control of uncertain mechanical systems with actuator faults: a sliding mode observer-based approach," IEEE Transactions on Control Systems Technology, vol. 26, no. 4, pp. 1249-1258, 2018.

[16] Y. Sun, Z. Zhang, M. Leibold et al., "Protective control for robot manipulator by sliding mode based disturbance reconstruction approach," in Proceedings of the 2017 IEEE International Conference on Advanced Intelligent Mechatronics (AIM), pp. 1015-1022, Munich, Germany, 2017.

[17] W. Liu and P. Li, "Disturbance observer-based fault-tolerant adaptive control for nonlinearly parameterized systems," IEEE Transactions on Industrial Electronics, vol. 66, no. 11, pp. 8681-8691, 2019.

[18] C.-C. Chen, S. S.-D. Xu, and Y.-W. Liang, "Study of nonlinear integral sliding mode fault-tolerant control," IEEE/ASME Transactions on Mechatronics, vol. 21, no. 2, pp. 1160-1168, 2016.

[19] Y.-W. Liang, C.-C. Chen, D.-C. Liaw, and Y.-T. Wei, "Nonlinear reliable control with application to a vehicle antilock brake system," IEEE Transactions on Industrial Informatics, vol. 9, no. 4, pp. 2114-2123, 2013.

[20] S. M. Smaeilzadeh and M. Golestani, "Finite-time fault-tolerant adaptive robust control for a class of uncertain nonlinear systems with saturation constraints using integral backstepping approach," IET Control Theory \& Applications, vol. 12, no. 15, pp. 2109-2117, 2018.

[21] A. Mohammadi, M. Tavakoli, H. J. Marquez, and F. Hashemzadeh, "Nonlinear disturbance observer design for robotic manipulators," Control Engineering Practice, vol. 21, no. 3, pp. 253-267, 2013.

[22] W. Liang, S. Huang, S. Chen, and K. K. Tan, "Force estimation and failure detection based on disturbance observer for an ear surgical device," ISA Transactions, vol. 66, pp. 476-484, 2017.

[23] B. Xiao and S. Yin, "Velocity-free fault-tolerant and uncertainty attenuation control for a class of nonlinear systems," IEEE Transactions on Industrial Electronics, vol. 63, no. 7, pp. 4400-4411, 2016.

[24] M. W. Spong, S. Hutchinson, and M. Vidyasagar, Robot Modeling and Control, Wiley, New York, NY, USA, 2006. 